\title{
On the Upper Critical Dimension of Bernoulli Percolation
}

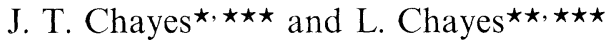

Laboratory of Atomic and Solid State Physics, Cornell University, Ithaca, NY 14853, USA

Abstract. We derive a set of inequalities for the
percolation problem. Assuming the existence
inequalities imply:
\[ \begin{array}{l}f+v \geqq 1+\beta_{Q}, \\ \mu+v \geqq 1+\beta_{Q}, \\ \zeta \geqq \min \left\{1, \frac{v^{\prime}}{v}\right\},\end{array} \]

where the above exponents are $f$ : the flow constant exponent, $v\left(v^{\prime}\right)$ : the correlation length exponent below (above) threshold, $\mu$ : the surface tension exponent, $\beta_{Q}$ : the backbone density exponent and $\zeta:$ the chemical distance exponent. Note that all of these inequalities are mean-field bounds, and that they relate the exponent $v$ defined from below the percolation threshold to exponents defined from above threshold. Furthermore, we combine the strategy of the proofs of these inequalities with notions of finite-size scaling to derive:

$$
\max \left\{d v, d v^{\prime}\right\} \geqq 1+\beta_{Q},
$$

where $d$ is the lattice dimension. Since $\beta_{Q} \geqq 2 \beta$, where $\beta$ is the percolation density exponent, the final bound implies that, below six dimensions, the standard order parameter and correlation length exponents cannot simultaneously assume their mean-field values; hence an implicit bound on the upper critical dimension: $d_{c} \geqq 6$.

\footnotetext{
* Work supported by the U.S. Army Research Office through the Mathematical Sciences Institute of Cornell University

$\star \star$ Work supported by the DOE under Grant No. DE-AC01-83ER13044

$\star \star \star$ Current address: Department of Mathematics, University of California, Los Angeles, CA 90024 , USA
} 


\section{Introduction}

The upper critical dimension of a system is the dimension above which its critical behavior can be described by mean-field theory. Indeed, if one calculates exponents in a mean-field (or Bethe lattice) theory and substitutes these exponents into hyperscaling relations (relations between exponents which involve the spatial dimension), the upper critical dimension is obtained by solving for dimension as the unknown. More sophisticated treatments, based on the renormalization group approach to "equivalent" field theories, generally yield satisfactory agreement with this procedure.

The first rigorous proof of mean-field behavior was the work of Aizenman [A] and Fröhlich [F], who showed, among other results, that in Ising-like systems, the susceptibility exponent, $\gamma$, assumes its mean-field value in dimension exceeding four. (See also $[\mathrm{AG}, \mathrm{AF}, \mathrm{FS}]$ for improvements and some refinements at $d=4$.) The basic ingredient in these proofs is the question of convergence, divergence or marginality of integrals of products of two-point functions. Thus, if the two-point function tends to zero as $\left\langle\sigma_{0} \sigma_{x}\right\rangle \sim|x|^{-(d-2+\eta)}$ at the critical point, one has $\gamma=1$ whenever $d+2 \eta-4>0$. Since, by the bounds of [FSS], one has $\eta \geqq 0$ in all dimensions, there is mean-field behavior provided $d>4$.

A similar approach to percolation has met with only limited success. In [AN], the relevant diagrammatics were identified, the result being that $\gamma=1$ whenever $d+3 \eta-6>0$. However, no a priori lower estimate on $\eta$ was available for this system. Worse yet, the $\varepsilon$-expansion shows that to low order, $\eta$ is actually negative [PL, AKM], and high-density series are consistent with $\eta \leqq 0$ in $d=3$ [GS].

Since establishing useful bounds on $\eta$ for percolation problems in any dimension (other than two) seems almost hopeless at the present time, one might pose the less ambitious question: How low must the dimension be to force nonmean-field behavior in percolation?

For two-dimensional percolation, non-mean-field behavior can be explicitly demonstrated with upper and/or lower bounds on various exponents [K 1]. In $d>2$, results have been somewhat limited. Based on finite-size scaling, the bound $v \geqq \frac{2}{d}$ was obtained in [CCFS] for the correlation length exponents in a general class of disordered systems which includes percolation. One direct consequence of this bound is that $v$ cannot assume its mean-field value (of $\frac{1}{2}$ ) when $d<4$. Unfortunately, the arguments used in [CCFS] did not distinguish percolation from the other systems to which the results apply. The price of this generality is of course reflected in the poor estimate of an upper critical dimension for percolation - that is, $d_{c} \geqq 4$.

In this work, we will derive inequalities for percolation which (if various critical exponents exist) imply

$$
\max \left\{d v, d v^{\prime}\right\} \geqq 1+2 \beta,
$$

where $v$ and $v^{\prime}$ are the correlation length exponents above and below threshold and $\beta$ is the order parameter (percolation density) exponent.

If, as in the analysis of mean-field theories, one substitutes the mean-field values of $\beta=1$ and $v^{\prime}=v=\frac{1}{2}$ into (1), and solves for dimension, the result is $d \geqq 6$. Explicitly, (1) implies that the correlation length and order parameter exponents cannot simultaneously assume their mean-field values when the dimension is lower than six. 
Some amusing features of (1) are:

i) (1) relates critical exponents from above and below the percolation threshold, and

ii) (1) involves the explicit (additive) appearance of a constant.

As will become clear from the derivation, (i) and (ii) are closely related.

In fact, (1) represents the end product of several related inequalities, and is not the first item on the agenda:

After a brief (but inevitable) section in which the relevant definitions are reviewed, in Sect. 2 we will derive what is perhaps the fundamental inequality of this note:

$$
f+v \geqq 1+\beta_{Q} .
$$

Here $f$ is the flow capacitance exponent and $\beta_{Q}$ the backbone density exponent.

In Sect. 3, we will describe surface tension from a finite-size scaling perspective and show that in (2), the exponent $f$ can be replaced by the surface tension exponent, $\mu$, to obtain

$$
\mu+v \geqq 1+\beta_{Q} .
$$

In Sect. 4, we will supplement the derivations of previous sections to obtain the inequality (1). The necessary ingredient is an acceptable definition of the correlation length, $\xi^{\prime}$, in the percolating regime. We therefore discuss in some detail the current status of the rigorous work on $\xi^{\prime}$, and introduce a (superfluous but aesthetically pleasing) finite-size scaling $\xi^{\prime}$ from which the inequality (1) is easily derived.

Finally, in Sect. 5, we will quickly define a chemical distance exponent, $\zeta$, and show that all our previous considerations are actually a gilded version of a (rather trivial) mean-field inequality for $\zeta$ :

$$
\zeta \geqq \min \left\{1, \frac{v^{\prime}}{v}\right\} .
$$

\section{Notation and Definitions}

We will attempt to be as brief as possible; the reader is urged to skip this section and refer back to it if necessary. More background details and further discussion can be found in [CC1].

The percolation problems we consider will take place on the unit-spaced $d$-dimensional hypercubic lattices $\mathbb{Z}^{d}$. The nearest neighbor pairs of $\mathbb{Z}^{d}$ will be called bonds. Bonds are said to be connected if they share a site.

Percolation problems are defined by choosing a $p \in(0,1)$ and declaring each bond to be "occupied" (or "vacant") with independent probability $p$ (or $1-p$ ). Occupied bonds may be visualized by drawing a line segment connecting the endpoints. The event that a given bond fails to be occupied may be visualized in one of two equivalent ways: no line segment is drawn, or the $(d-1)$-cell orthogonal to the vacant bond whose center lies at the center of the (missing) line segment is declared to be occupied. These orthogonal cells are called dual $(d-1)$-cells (bonds 
in $d=2$, plaquettes in $d=3, \ldots)$, and live on the dual lattice $\left(\mathbb{Z}+\frac{1}{2}\right)^{d}$. Events and configurations of occupied bonds always admit an equivalent description in terms of these dual cells.

The (product) measure at parameter value $p$ on the set of all possible bond configurations will be denoted by $\operatorname{Prob}_{p}(-)$ or $\mu_{p}(-)$, and is called Bernoulli measure at density $p$. More generally, expectations with respect to these measures will be denoted by $E_{p}(-)$.

It is known [BH] that if $d \geqq 2$, there is a $p_{c} \in(0,1)$ such that, with probability one, whenever $p>p_{c}$, there is an infinite connected cluster of occupied bonds, while if $p<p_{c}$, all bonds belong to clusters of finite size. The value $p_{c}$ is called the percolation threshold. We will denote by $P_{\infty}(p)$ the probability that a bond belongs to an infinite cluster. If both ends of a bond belong to separate (disjoint) infinite occupied clusters - that is, clusters which need not both make use of any given occupied bond - the bond is said to belong to the backbone of the infinite cluster. We will denote the backbone (bond) density by $Q_{\infty}(p){ }^{1}$

For $p<p_{c}$, the probability that two distant sites belong to the same connected cluster of occupied bonds tends to zero. The correlation length below $p_{c}$ is defined by considering $\tau_{\text {on }}(p)$, the probability that the origin and $(n, 0, \ldots, 0)$ are connected by a path of occupied bonds. It turns out that $\left[\tau_{\text {on }}(p)\right]^{1 / n}$ converges to a limit which is denoted by $e^{-1 / \xi(p)}$. The quantity $\xi(p)$ is called the correlation length. It has been shown [AB, MMS] that for $p<p_{c}, \xi(p)$ is finite, diverging continuously as $p \uparrow p_{c}$. Above $p_{c}$, a different notion of a correlation length must be introduced [since $\xi(p)$ is identically infinite]. This will be discussed in Sect. 4.

It is a widely held belief that, in these problems, $P_{\infty}(p), Q_{\infty}(p)$, and $\xi(p)$ are smooth functions of $p$, vanishing or diverging at $p_{c}$ with a power law. Other quantities of interest ("scaling quantities") are also believed to have power laws at the critical point. Various aspects of this issue have been established under certain conditions - which we will not attempt to list. It is worth stating that these questions are, to the largest extent, open.

Most of the results in this paper amount to bounds relating various scaling quantities. Thus, if the scaling quantities have power laws (critical exponents), the bounds imply inequalities between these exponents. We will use the symbols $\beta$ and $\beta_{Q}$ for the exponents with which the percolation and backbone density vanish. The letter $v$ is used for the exponent with which the correlation length diverges. Other exponents will be introduced as necessary.

We will make no attempt to interpret for the reader the sense in which the exponent inequalities hold; we will assume that those with the inclination to do so can, e.g. take logs and inferior or superior limits.

We will often be interested in the statistical behavior of configurations in (large) finite rectangles. We will thus denote by $\Lambda_{L}$ the set of sites

$$
\Lambda_{L}=\left\{x \in \mathbb{Z}^{d} \mid 0 \leqq x_{1}, \ldots, x_{d} \leqq L\right\},
$$

\footnotetext{
${ }^{1}$ The notation $P_{\infty}(p)$ and $Q_{\infty}(p)$ are often reserved for the probability that a given site (e.g. the origin) is a part of the infinite cluster or the backbone. It is clear that (when positive) the ratio of the associated bond or site probabilities are bounded above and below by uniform constants
} 
or, perhaps, the set of bonds with both endpoints satisfying the above restriction. Embarrassingly enough, many results connected with the critical behavior in finite regions - which ought to hold in cubes - have only been rigorously demonstrated in more hospitable rectangles. We therefore introduce the notation

$$
\Lambda_{n, L}=\left\{x \in \mathbb{Z}^{d} \mid 0 \leqq x_{1}, \ldots, x_{d-1} \leqq n L, 0 \leqq x_{d} \leqq L\right\},
$$

where $n>1$ is an integer, more often than not equal to three. The size of these rectangles, as defined by the number of bonds, will be indicated with vertical bars, e.g.

$$
\left|\Lambda_{L}\right|=\text { (number of bonds with both endpoints in } \Lambda_{L} \text { ). }
$$

For future reference, the boundary of these objects will be denoted by e.g. $\partial \Lambda_{n, L}$ :

$$
\partial \Lambda_{n, L}=\left\{x \in \Lambda_{n, L} \mid x \text { has a neighbor in } \mathbb{Z}^{d} \backslash \Lambda_{n, L}\right\} .
$$

Many results which are believed to be true whenever $p>p_{c}$ have only been established under technically stronger hypotheses (or in two dimensions). The "usual" assumption is as follows: Consider the lattice $\left(\mathbb{Z}^{+}\right)^{2} \times \mathbb{K}^{(d-2)}$, where $\mathbb{Z}^{+}=\{x \in \mathbb{Z} \mid x \geqq 0\}$ and $\mathbb{K}=\{x \in \mathbb{Z} \mid 0 \leqq x \leqq k\}$. Denote by $\hat{p}_{c}^{(k)}$ the percolation threshold in these (quadrant) layers, and let

$$
\hat{\varrho}_{c}=\lim _{k \rightarrow \infty} p_{c}^{(k)} \text {. }
$$

(The $p_{c}^{(k)}$ form a monotone sequence, so the limit always exists.) Clearly $\hat{\varrho}_{c} \geqq p_{c}$, although one believes that $\hat{\varrho}_{c}=p_{c}$ in all dimensions. Unfortunately, most of the important quantities that we will consider above threshold (the flow capacitance, the correlation length and the surface tension) have actually been established to be non-trivial only for $p>\hat{\varrho}_{c}$. Establishing the equality of $\hat{\varrho}_{c}$ and $p_{c}$ is, in our minds, one of the more important open problems in this subject.

\section{Flow Capacitance}

The flow capacitance problem has been described in a variety of contexts $[\mathrm{K} 2, \mathrm{~K} 3$, CC 1, CC 2]. Despite its somewhat unphysical character, it is a pleasant, analyzable model which describes bulk transport in a random media. Roughly speaking, the occupied bonds of the Bernoulli problem are considered to be open channels, while the vacant ones are blocked. Fluid is allowed to flow down the open channels at maximum flux of unity, subject to the constraint of current conservation at the sites.

The problem is generally studied in large finite squares or rectangles, e.g. $\Lambda_{L}$ as defined in Sect. 1. If $\omega$ is a configuration of occupied bonds in $\Lambda_{L}$ in which the top face $\left(\left\{x \in \Lambda_{L} \mid x_{d}=L\right\}\right)$ is disconnected from the bottom, then there is no flow through the cube. On the other hand, if there is an open channel connecting top and bottom, the removal of a few key bonds could disconnect these faces. How much fluid can flow through the cube is determined by how few bonds must be removed in order to disconnect a chosen pair of opposing faces. We thus define $\Phi_{L}(\omega)$ to be the minimum number of bonds in $\omega$ whose removal would disconnect 
the top face of $\Lambda_{L}$ from the bottom. Alternatively:

$$
\Phi_{L}(\omega)=\left(\# \text { of disjoint crossings from top to the bottom of } \Lambda_{L} \text { in } \omega\right) \text {. }
$$

When we count the number of disjoint channels which connect the two near faces of $A_{n, L}$, we will use the notation $\Phi_{n \cdot L}$.

The flow capacitance of the medium is determined by the large $L$ behavior of $\frac{\Phi_{L}}{L^{d}-1}$. Here we must proceed with a little caution: There are several senses in which $L^{-(d-1)} \Phi_{L}$ could converge to a limit -- the nicest of which would be almost surely. The general problem, in $d \geqq 3$, of the large $L$ behavior of $\Phi_{L}$ is, at present, open. For $p<p_{c}$, it is easy to show that $\Phi_{L} \rightarrow 0$. For $p>\varrho_{c}$ it has been shown [CC 1, CC 2] that with probability tending to one, $\frac{\Phi_{L}}{L^{\frac{d-1}{d-}}}$ is bounded below uniformly for $L$ large enough. Under the condition $(1-p) \ll 1$, Kesten $[\mathrm{K} 2, \mathrm{~K} 3]$ has established essentially optimal results including the a.s. existence of the $L \uparrow \infty$ limit of $\Phi_{n, L} /[n L]^{d-1}$ independent of $n .^{2}$

We will not address the issue of the existence of limits in this note. Instead, we first define the median flow

$$
\widetilde{\Phi}_{L}=\max \left\{n \mid \Phi_{L}>n \text { with Prob }>\frac{1}{2}\right\}
$$

and

$$
\tilde{\varphi}_{L} \equiv L^{-(d-1)} \widetilde{\Phi}_{L}
$$

We will work with the infinite-volume flow capacitance

$$
\tilde{\varphi}_{*}=\limsup _{L \rightarrow \infty} \tilde{\varphi}_{L}
$$

When the need arises, we will use similar notation amended by subscripted $n$ 's to denote flow capacitances in boxes of other shapes.

The $\tilde{\varphi}_{*}(p)$ are monotone increasing and vanish for $p<p_{c}$. Presumably, they are zero at $p_{c}$ and positive for $p>p_{c}$, but this has not yet been rigorously established. Under the optimistic assumption that $\tilde{\varphi}_{*}(p)$ vanishes at $p_{c}$ with a power law, we will denote the power by $f$.

Before we get to the principal business of this section, we need the following straightforward result:

Lemma 1. Let $Q_{n}(p)=n^{-d} E_{p}$ (\# of bonds in $\Lambda_{n}$ which are connected to $\partial_{n}$ by two disjoint paths). Then, if $p>p_{c}$,

$$
\lim _{n \rightarrow \infty} Q_{n}(p)=Q_{\infty}(p)
$$

Proof. If a bond, $b \in A_{n}$, is in the backbone, it is connected by two disjoint paths of occupied bonds to the boundary $\partial \Lambda_{n}$. This implies the bound

$$
Q_{n}(p) \geqq Q_{\infty}(p) .
$$

\footnotetext{
${ }^{2}$ In fact, one can even consider the flow in drastically elongated shapes. Provided that the crosssectional area is a (small) constant times the log of the length in the flow direction, the results of $[\mathrm{K} 2, \mathrm{~K} 3]$ and [CC 1, CC 2] apply
} 
Other mechanisms by which $b$ may be connected to the boundary include $b$ being in a finite cluster or in a "dangling end." (These are the only possibilities; there are no "spineless clusters, "since we have assumed that $p>p_{c}$. For a detailed discussion of this and other points in this paragraph, see [CC $3, \mathrm{CC} 4]$.) If $b$ is in a dangling end, there is a unique bond which attaches $b$ to the backbone; furthermore, the removal of this bond would place $b$ in a finite cluster. Thus both of the above mechanisms, in essence, imply that $b$ is in a finite cluster which is attached to the boundary.

Pick $k \ll n$ and denote by $\varepsilon_{k}(p)$ the probability that a bond is in a finite cluster or dangling end of size exceeding $k$. It is not hard to see that

$$
Q_{n}(p) \leqq Q_{\infty}(p)+\varepsilon_{k}(p)+(\text { const }) 2 d \frac{k}{n} .
$$

The desired equality follows by taking first $n$ and then $k$ to infinity.

We are now prepared for the principal result of this section.

Theorem 2. Let $\varepsilon>0$ and define the below-threshold correlation length length $\xi(\varepsilon)$ $\equiv \xi\left(p_{c}-\varepsilon\right)$, and the (above-threshold) flow capacitance $\tilde{\varphi}_{*}(\varepsilon) \equiv \tilde{\varphi}_{*}\left(p_{c}+\varepsilon\right)$ and backbone density $Q_{\infty}(\varepsilon) \equiv Q_{\infty}\left(p_{c}+\varepsilon\right)$. Then

$$
\xi(\varepsilon) \geqq[\text { const }] \frac{\tilde{\varphi}_{*}(\varepsilon)}{\varepsilon Q_{\infty}(\varepsilon)} .
$$

Proof. Let $L \gg 1$ and consider the event $\mathscr{R}_{L}$ that two chosen opposing faces of $\Lambda_{L}$ are connected by a path of occupied bonds which lies inside $\Lambda_{L}$.

Our proof, which we will first run through informally, will amount to a lower bound on $e^{-L / \xi(\varepsilon)} \approx \operatorname{Prob}_{p_{c}-\varepsilon}\left[\mathscr{R}_{L}\right]$.

We begin with the observation that although the event $\mathscr{R}_{L}$ rarely occurs at $p_{c}-\varepsilon$, it is very likely at $p_{c}+\varepsilon$. Indeed, above threshold, one typically sees $\widetilde{\Phi}_{L}$ disjoint occurrences of $\mathscr{R}_{L}$. Since, roughly speaking, the only bonds which could contribute to the flow are those in the infinite backbone, one can expect to find a short path of length $t_{s} \lesssim \frac{L Q_{\infty}(\varepsilon)}{\tilde{\varphi}_{L}}$ crossing the box.

Now to get from $p_{c}+\varepsilon$ to $p_{c}-\varepsilon$, we take occupied bonds in the density $p_{c}+\varepsilon$ configuration and remove them with (independent) probability $\lambda(\varepsilon) \equiv \frac{2 \varepsilon}{p_{c}+\varepsilon}$. The probability that the short path will survive this removal is

$$
\left.(1-\lambda(\varepsilon))^{t_{\circ}} \geqq \exp [- \text { (const })\left(\varepsilon \frac{L Q_{\infty}(\varepsilon)}{\tilde{\varphi}_{L}}\right)\right] .
$$

Thus, as $L \rightarrow \infty$, one obtains

$$
\zeta(\varepsilon) \geqq[\text { const }] \frac{\tilde{\varphi}_{*}(\varepsilon)}{\varepsilon Q_{\infty}(\varepsilon)} .
$$

Let us now attend to details. For the lower bound on $\operatorname{Prob}_{p_{c}-\varepsilon}\left[\mathscr{R}_{L}\right]$, divide $\Lambda_{L}$ and its immediate vicinity into $N$ cubes, $\Lambda_{n}^{(i)}, i=1, \ldots, N$, of scale $n$ with $n$ fixed and 
$N=(L / n)^{d}\left(1+O\left(L^{-1}\right)\right)$ chosen so that $\Lambda_{\bar{L}}=\bigcup_{i=1}^{N} \Lambda_{n}^{(i)}$ contains $\Lambda_{L}$. Let $\Omega_{\bar{L}}$ denote the set of configurations of the slightly larger cube. If $\omega \in \Omega_{\bar{L}}$, define $Q_{n}^{(i)}(\omega)$ by

$$
Q_{n}^{(i)}(\omega)=\left(\# \text { of bonds in } \Lambda_{n}^{(i)} \text { connected to } \partial \Lambda_{n}^{(i)} \text { by two disjoint paths }\right)
$$

It is evident that the $Q_{n}^{(i)}(\omega)$ are i.i.d. random variables with mean $Q_{n}(\varepsilon) \equiv Q_{n}\left(p_{c}+\varepsilon\right)$.

Next, define $\Omega_{\Phi_{L}}=\left\{\omega \in \Omega_{\bar{L}} \mid \Phi_{L}(\omega) \geqq \widetilde{\Phi}_{L}(\varepsilon)\right\} \quad$ [so that, by definition, $\left.\mu_{p_{c}+\varepsilon}\left(\Omega_{\Phi_{L}}\right) \geqq \frac{1}{2}\right]$. It is clear that, for each $\omega \in \Omega_{\Phi_{L}}$, one can find an occupied path crossing $\Lambda_{L}$ of length $\ell_{s}(\omega)$, where

$$
\ell_{\jmath}(\omega) \leqq \frac{1}{\widetilde{\Phi}_{L}(\varepsilon)} n^{d} \sum_{i=1}^{N} Q_{n}^{(i)}(\omega) .
$$

Note that the fact that we are above threshold is enough to ensure that we are not dividing by zero in the right-hand side of (18). Indeed, if $p>p_{c}$, the surface of a cube of scale $K$ is, by the ergodic theorem, connected to infinity with probability tending to one as $K \uparrow \infty$. Thus, in cubes of scale $L \gg K \gg 1$, there are, with high probability, paths between opposing faces of $\Lambda_{L}$ which go to the surface of a smaller cube, of scale $K$, located in the center. Using the recent result of uniqueness of the infinite cluster above threshold [AKN], one can therefore find an intermediate scale, $K^{*}$, inside of which it is very likely that these paths will connect. Thus, one has

$$
\lim _{L \rightarrow \infty} \operatorname{Prob}_{p_{c}+\varepsilon}\left(\Phi_{L} \geqq 1\right)=1 .
$$

Using the law of large numbers we have that, for any positive $\delta$,

$$
\operatorname{Prob}_{p_{c}+\varepsilon}\left[\sum_{i} Q_{n}^{(i)} \geqq(1+\delta) N Q_{n}(\varepsilon)\right]
$$

tends to zero. Thus, for $N$ large enough, $\sum_{i} Q_{n}(i)<(1+\delta) N Q_{n}$ with probability exceeding $\frac{3}{4}$, and hence, for all sufficiently large $L$,

$$
\mu_{p_{c}+\varepsilon}\left(\Omega_{\Phi_{L}} \cap\left\{\sum_{i} Q_{n}^{(i)}<(1+\delta) N Q_{n}\right\}\right) \geqq \frac{1}{4} .
$$

As $L \uparrow \infty$, we may therefore write

$$
\operatorname{Prob}_{p_{c}-\varepsilon}\left[\mathscr{R}_{L}\right] \geqq \frac{1}{4} \exp \left[-c \varepsilon N(1+\delta) n^{d} \frac{Q_{n}(\varepsilon)}{\widetilde{\Phi}_{L}(\varepsilon)}\right],
$$

where $c=O(1)$ is any constant for which $(1-\lambda(\varepsilon)) \geqq e^{-\varepsilon c}$ uniformly in $\varepsilon$.

Next, we rewrite $\tilde{\Phi}_{L}(\varepsilon) \equiv \tilde{\varphi}_{L}(\varepsilon) L^{d-1}$ and $n^{d} N=L^{d}\left(1+O\left(\frac{1}{L}\right)\right)$, and take the $\left(\frac{1}{L}\right)^{\text {th }}$ root of both sides of (21). Using the known result that $\left[\operatorname{Prob}_{p_{c}-\varepsilon}\left[\mathscr{R}_{L}\right]\right]^{1 / L}$ $\rightarrow e^{1 / \xi}$ [see, e.g., Eq. (46)] and letting $L$ tend to infinity along any sequence which produces $\tilde{\varphi}_{*}(\varepsilon)$, we obtain

$$
\frac{1}{\xi(\varepsilon)} \leqq c(1+\delta) \frac{\varepsilon Q_{n}(\varepsilon)}{\tilde{\varphi}_{*}}
$$

We now let $n \uparrow \infty$ and, if desired, discard $\delta$. The final result, as promised, is

$$
\xi(\varepsilon) \geqq c \frac{\tilde{\varphi}_{*}(\varepsilon)}{\varepsilon Q_{\infty}(\varepsilon)} .
$$


Corollary. If, as $p \uparrow p_{c}$, the correlation length diverges with exponent $v$, while as $p \downarrow p_{c}$, the backbone density vanishes with exponent $\beta_{Q}$ and the flow capacitance vanishes with exponent $f$, then

$$
f+v \geqq 1+\beta_{Q} .
$$

Remark. As noted earlier, the inequality in Theorem 2 relates quantities defined above and below the percolation threshold, in particular at equal distances, $\varepsilon$, above and below $p_{c}$. However, a quick glance at the proof shows that one need not evaluate the relevant quantities at equal distances above and below $p_{c}$, although this usually yields the most efficient bound. Indeed, suppose that the distance below the critical point is $\varepsilon$ while the distance above is $\varepsilon^{\prime}$. Setting $\varepsilon=\left(\varepsilon^{\prime}\right)^{b}$ with $b \geqq 1$, the result is $f+b v \geqq 1+\beta_{Q}$, which is not particularly enlightening. On the other hand, if we set $\varepsilon^{\prime}=\varepsilon^{a}$ with $a \gg 1$ we get

$$
f \geqq \beta_{Q} .
$$

This rather simple minded bound on $f$ (noted in [CC 2]) actually turns out to be more efficient than (2) in $d=2$.

\section{Surface Tension}

The subject of surface tension for Bernoulli systems in dimension three and higher was addressed some time ago in [ACCFR]. Details and definitions can be found in [ACCFR] and/or [CC 1]. For our purposes, the $(d=3)$ problem is formulated as follows:

Consider the three-dimensional Bernoulli bond system at occupation density $p$. The plaquettes dual to vacant bonds may be regarded as occupied (with independent probability $1-p$ ). Now denote by $\gamma_{L}$ a square loop of side $L$ composed of dual lattice edges. The event of interest is:

$$
\mathscr{W}_{i L}=\left\{\omega \mid \exists \text { a collection of occupied plaquettes whose boundary is } \gamma_{L}\right\},
$$

in terms of which the surface (or string) tension may be defined as the limit:

$$
\sigma(p) \equiv \lim _{L \rightarrow \infty}-\frac{1}{L^{2}} \log E_{p}\left[\mathscr{W}_{\gamma_{L}}\right]
$$

The results of [ACCFR] are as follows: First (using the coincidence of critical points established in $[\mathrm{AB}, \mathrm{MMS}])$, it was shown that below $p_{c}, \sigma(p)$ vanishes. Furthermore, when $p$ exceeds the critical point $\hat{\varrho}_{c}$ (which we remind the reader morally coincides with $\left.p_{c}\right), \sigma(p)$ is positive.

The expected behavior of $\sigma(p)$ is that it vanishes at threshold according to a power law; the purported critical index will be denoted by $\mu$.

[ACCFR] also studied a generalization of the problem of plaquettes spanning loops in three dimensions to $r$-cells spanning $(r-1)$-cell loops in $d>r$ dimensions. In particular, for the case $r=(d-1)$, i.e. the problem dual to bonds, the principal results cited above hold mutatis mutandis. Since this is the case that concerns us here, we will write $\sigma(p)$ and $\mu$ in reference to the $(d-1)$-cell surface tension (not the two-cell surface tension). 
The idea of surfaces spanning loops arises in the context of field theoretic problems. From the view-point of statistical mechanics, one is interested in interfaces across large hypercubes (e.g. our standard $\Lambda_{L}$ ), that is, interfaces which are not pinned to any loop, but which must remain within a hypercube. Such surfaces have been investigated by Kesten [K 2, K 3]. Let us introduce some notation to study such interfaces:

Let $B_{L, k}$ denote the box

$$
B_{L, k}=\left\{x \in \mathbb{Z}^{d} \mid 0 \leqq x_{1}, \ldots, x_{d-1} \leqq L ; 0 \leqq x_{d} \leqq k\right\}
$$

and

$$
\mathscr{I}_{L, k}=\left\{\omega \mid \exists \text { an interface in } B_{L, k} \text { which separates } x_{d}=0 \text { from } x_{d}=k\right\} .
$$

Note that the event $\mathscr{I}_{L, k}$ implies (or is defined by) the condition that no point on the $x_{d}=0$ face is connected to the face at $x_{d}=k$ by occupied bonds inside $B_{L, k}$. We further define

$$
I_{L, k}(p) \equiv \operatorname{Prob}_{p}\left[\mathscr{I}_{L, k}\right] .
$$

The event $\mathscr{I}_{L, k}$ is depicted in Fig. 1 below

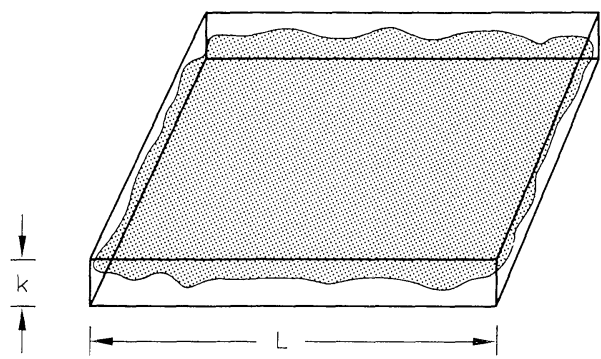

Fig. 1. The event $\mathscr{I}_{L, k}$

These interfaces suggest the following notion of surface tension:

Proposition 3. The limits

$$
\sigma_{k} \equiv \lim _{L \rightarrow \infty}-\frac{1}{L^{d-1}} \log I_{L . k}
$$

exist for each $k$. Furthermore, $I_{L, k}$ obeys the a priori lower bound

$$
I_{L, k} \geqq e^{-\sigma_{k} L^{d-1}},
$$

while if ( say) $L>3 k$, one can find a constant $c$ (which will depend on dimension) such that

$$
I_{L, k} \leqq e^{-c \sigma_{k}} L^{d-1}
$$

Finally, the limit of the sequence $\left\{\sigma_{k}\right\}$ exists and is, in fact, equal to the full-space surface tension defined in Eq. (26). 


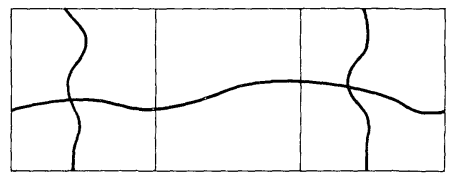

Fig. 2. Block cell event

Proof. It is not hard to see that for any (integer) $n$,

$$
I_{n L, k} \leqq\left[I_{L, k}\right]^{n^{d-1}},
$$

which, together with the typical subadditive arguments, both establishes existence of the limits

$$
\sigma_{k}=\lim _{L \rightarrow \infty}-\frac{1}{L^{d-1}} \log I_{L, k},
$$

and proves the a priori bound. It goes without saying that for finite $k, \sigma_{k}>0$.

Next, observe that if $L$ is on the scale of $k$ (e.g. $L=3 k$ ), block cells can be constructed out of the event $\mathscr{I}_{L, k}$ and pieces of translations thereof (see Fig. 2 above). Block events of this sort were used in various stages of [ACCFR]; the details can be found in Sect. 3 ii.) These $3^{d-1} \times 1$ block events can be shown to occur with probability larger than $\left[I_{3 k, k}\right]^{\left[1+\frac{2 d}{3}\right]}$. Moreover, the block events have desirable connectivity properties, and can therefore be used to construct yet larger interfaces. Indeed, the event $\mathscr{I}_{N k, k}$ can be produced with probability exceeding $\left[I_{3 k, k}\right]\left[1+\frac{2 d}{3}\right] N^{d-1}$. This, together with the existence of $\sigma_{k}$, implies that $\log \left[I_{3 k, k}\right]$ $\geqq c \sigma_{k} k^{d-1}$.

Finally, let us attend to the large $k$ behavior of the $\sigma_{k}$. First observe that the $\left\{\sigma_{k}\right\}$ form a monotone sequence:

$$
\sigma_{k+1} \leqq \sigma_{k}
$$

implying the existence of a limit which we will temporarily denote by $\sigma_{\infty}$. It is clear that $\sigma_{\infty} \geqq \sigma$. Indeed, for any $k$, we have

$$
E_{p}\left[\mathscr{W}_{\gamma_{L}}\right] \geqq I_{L . k}(p)[1-p]^{2(d-1) k L^{d-2}},
$$

since plating of the walls of $B_{L, k}$, together with the event $\mathscr{I}_{L, k}$, produces the event $\mathscr{W}_{\gamma_{L}}$. This means that $\forall k, \sigma_{k} \geqq \sigma$.

Next, recalling that the limit of $-\frac{1}{L^{d-1}} \log E_{p}\left[\mathscr{W}_{\nu_{L}}\right]$ actually exists, it is clear that for fixed $\varepsilon$, we can find $L$ large enough so that

$$
E_{p}\left[\mathscr{W}_{\gamma_{L}}\right] \geqq e^{-[\sigma+(1 / 2) \varepsilon] L^{d-1}} .
$$

Consider the event $\mathscr{W}_{\gamma_{L} \mid H}, H>L$, that in the hypercube of side $H$ with $\gamma_{L}$ at its center, we can find a surface spanning $\gamma_{L}$. Since $\mathscr{W}_{\gamma_{L} \mid H_{2}} \supset \mathscr{W}_{\gamma_{L} \mid H_{1}}$ when $H_{1}>H_{2}$, and since $\mathscr{W}_{\gamma_{L}}=\bigcap_{H} \mathscr{W}_{\gamma_{L} \mid H}$, we have that for $H$ large enough

$$
E_{p}\left[\mathscr{W}_{\gamma_{L} \mid H}\right] \geqq e^{-[\sigma+\varepsilon] L^{d-1}}
$$


By plating together $N^{d-1}$ translates of $\gamma_{L}$, and insisting that the event $\mathscr{W}_{\gamma_{L} \mid H}$ occur for each of these loops, we have (modulo relatively insignificant boundary events) created an interface in $B_{N L+(1 / 2) H, H}$. Explicitly

$$
\mathscr{I}_{N L+(1 / 2) H, H} \geqq\left(e^{-[\sigma+\varepsilon][N L]^{d-1}}\right)\left([1-p]^{H^{d}[N L]^{d-2}}\right),
$$

where, to obtain the last factor, we have (wastefully) insisted all the cubes along the perimeter are devoid of bonds. Equation (36) implies the bound $\sigma_{I I} \leqq \sigma+\varepsilon$, which establishes $\sigma_{\infty}=\sigma$.

Although of no immediate use, this finite scaling approach to surface tension yields some amusing corollaries.

Corollaries. (A) Let $\gamma_{L}$ denote the standard loop of scale $L$, and consider the following three events:

i) $\mathscr{W}_{\gamma_{L} ; \|}: \gamma_{L}$ is spanned by a surface in the region $\left|x_{1}\right|, \ldots,\left|x_{d-1}\right| \leqq L$.

ii) $\mathscr{W}_{\gamma_{L} ;-}: \gamma_{L}$ is spanned by a surface in the half-space.

iii) $\mathscr{W}_{\gamma_{L} ; 0}: \gamma_{L}$ is spanned by an oriented surface.

Then all of these events have associated surface tensions, which we denote by $\sigma_{\|}$, $\sigma_{-}$, and $\sigma_{0}$, respectively. However, $\left(\sigma_{\|}, \sigma_{-}, \sigma_{0}\right)=\sigma$ for all $p$.

(B) The surface tensionn $\sigma(p)$ is a right continuous function of $p$ i.e.

$$
\lim _{\varepsilon \downarrow 0} \sigma(p+\varepsilon)=\sigma(p) \text {. }
$$

Proof. (A) Existence of these surface tensions follows from the usual subadditive arguments. Furthermore, since all the above listed events are more restrictive than $\mathscr{W}_{\gamma_{L}}$, these surface tensions are at least as large as $\sigma$. However, for any value of $k$, $\left(\sigma_{\|}, \sigma_{-}, \sigma_{0}\right)$ must be smaller than $\sigma_{k}$, since the event $\mathscr{I}_{L, k}$ (together with some lower order plating) produces an oriented surface in the column swept out by $\gamma_{L}$ in the half-space above $\gamma_{L}$. Equality of $\left(\sigma_{\|}, \sigma_{-}, \sigma_{0}\right)$ and $\sigma$ is squeezed from the $k \uparrow \infty$ limit.

(B) Continuity of finite-volume probabilities can be easily exploited to establish continuity of $\sigma_{k}(p)$. This shows that $\sigma(p)$ is a decreasing limit of continuous increasing functions. It is therefore right continuous.

In the previous section, we derived an inequality which involved $\tilde{\varphi}_{*}, Q_{\infty}$, and $\xi$. In a different context, [CC1, CC2], the flow capacitance was shown to be controlled by the surface tension. However, this was accomplished by considering the flow through "thin" slabs (of the form $a L \times L^{d-1}$ with $a \ll 1$ ). Another consequence of Proposition 3 is a strengthening of this result:

Proposition 4. Let $p=p_{c}+2 \varepsilon$ and denote by $\Phi_{3, L}$ the (easy-way) flow through $\Lambda_{3 . L}$. Then

$$
\Phi_{3, L} \geqq[\text { const }] L^{d-1}|\log \varepsilon| \sigma\left(p_{c}+\varepsilon\right)
$$

with probability exceeding

$$
1-\exp \left[-(\text { const }) L^{d-1} \sigma\left(p_{c}+\varepsilon\right)\right] .
$$

Proof. The proof is essentially the same as that of [CC1, CC2], although here we use the finite-size scaling estimates of Proposition 3. First, we observe that at $p_{c}+\varepsilon$

$$
\operatorname{Prob}\left[\Phi_{3, L}>0\right]=1-I_{3 L, L} \geqq e^{-c \sigma_{L} L^{d-1}} .
$$


Hence, using [ACCFR, Lemma 4.2], we have that at $p_{c}+2 \varepsilon$

$$
\operatorname{Prob}\left[\Phi_{3, L}>N\right] \geqq 1-\varepsilon^{-N} e^{-c \sigma_{L}(\varepsilon) L^{d-1}} .
$$

By choosing

$$
N=\frac{(1 / 2) c \sigma_{L} L^{d-1}}{|\log \varepsilon|}
$$

and assuming that $L$ is largw enough so that

$$
\sigma_{L} \leqq 2 \sigma
$$

the desired result follows.

Corollary. If $\varepsilon$ is chosen so that $\sigma(\varepsilon)>0$, then

$$
\xi(\varepsilon) \geqq[\text { const }] \frac{|\log \varepsilon|}{\varepsilon} \frac{\sigma(\varepsilon)}{Q_{\infty}(2 \varepsilon)} .
$$

Thus, if the various critical exponents exist, we have

$$
\mu+v \geqq 1+\beta_{Q} .
$$

Proof. Recalling the proof of Theorem 2, it is clear that we could have used a $[3 L]^{d-1} \times L$ box, rather than the hypercube $\Lambda_{L}$. In particular, we would then have derived an estimate of $\ell_{s}(\omega)$ involving $\Phi_{3, L}(\omega)$. At this point, instead of using a median flow, one simply uses the surface tension estimate of the above proposition.

Remarks. 1) As in the Remark following Theorem 2 of Sect. 2, we may scale differently above and below threshold to obtain the result

$$
\mu \geqq \beta_{Q},
$$

which was also derived in [CC1, CC2].

2) The reader may wonder whether Eq. (3) could have been derived directly from Eq. (2) by bounding $f$ in terms of $\mu$. It turns out that, at present, there are some technical difficulties in establishing an upper bound of the form $\tilde{\varphi}_{*} \geqq[$ const $] \varphi_{*, n}$; thus one cannot make a definite statement relating $f$ (as defined by $\varphi_{*}$ ) to $\mu$. It is worth noting, however, that the $\varphi_{*, m}$ and the $\varphi_{*, n}$ are bounded above and below by multiples of each other whenever $m, n>1$. Thus any possible " $f_{n}$ 's" are all equal for $n>1$; their mutual "value" is smaller than $\mu$.

\section{Correlation Lengths Above Threshold}

Below $p_{c}$, "correlations" in percolation problems are determined by whether or not two sites are in the same cluster. Thus, we define

$$
\tau_{0, x}(p) \equiv \operatorname{Prob}_{p}(0 \text { and } x \text { are in the same cluster }) \text {. }
$$


Denoting by $n$ the point $(n, 0, \ldots, 0)$, subadditivity (from the $\mathrm{FKG}$ inequality) ensures that the limit

$$
\frac{1}{\xi(p)} \equiv \lim _{n \rightarrow \infty}-\frac{1}{|n|} \log \tau_{0, n}(p)
$$

exists; this defines the correlation length $\xi(p)$.

Above $p_{c}, \tau_{0, n}$ would tend, asymptotically, to a constant. Thus, in order to extract meaningful information, one must either reword the definition of "correlation" or subtract the asymptotic constant. To this end, we define

$$
\tau_{0, x}^{\prime}(p) \equiv \operatorname{Prob}_{p}(0 \text { and } x \text { are in the same finite cluster }),
$$

and

$$
\tau_{0, x}^{\prime \prime}(p) \equiv \tau_{0, x}-P_{\infty}^{2}(p) .
$$

One of the difficulties to be faced when $p$ exceeds threshold is the lack, at present, of the proof of a bona fide limit for $n^{-1} \log \tau_{0, n}^{\prime}$ or $n^{-1} \log \tau_{0, n}^{\prime \prime}$. Indeed, this problem has two facets: (a) Does the limit actually exist, and, if so, (b) What relationship does the limit bear to the finite- $n$ correlations? Notice that an unexpected answer to (a) or (b) implies the existence of more than one important length scale. We will discuss this issue at greater length below; however, it is worth remarking that the limits of $n^{-1} \log \tau_{0, n}^{\prime}$ and $n^{-1} \log \tau_{0, n}^{\prime \prime}$ have recently been shown to agree along any subsequence [CCGKS]. Since $\tau_{0, n}^{\prime}(p)$ is the only correlation relevant for this work, we will omit any future reference to the double-primed quantity.

Below threshold, one not only has the existence of $\xi$, as defined in (43), but also the bound

$$
\tau_{0, n} \leqq e^{-n / \xi},
$$

which holds uniformly in $n$. Thus the correlation length serves two roles. To underscore explicitly the problem above $p_{c}$, note that one can always define

$$
\frac{1}{\xi_{a}^{\prime}}=\liminf _{n \rightarrow \infty}-\frac{1}{n} \log \tau_{0, n}^{\prime},
$$

so that, eventually,

$$
\tau_{0, n}^{\prime} \leqq e^{-\frac{1}{2} n / \xi_{a}^{\prime}},
$$

(where the choice of 2 is of no particular significance). However, a relevant question which must be addressed is how large does $n$ have to be before (48) is valid? We must therefore consider the length

$$
\xi_{b}^{\prime}=\min _{k}\left\{\tau_{0, n}^{\prime} \leqq e^{-\frac{1}{2} n / \xi_{a}^{\prime}} \forall n>k\right\} .
$$

Now if it happens that $\xi_{b}^{\prime}$ exceeds $\xi_{a}^{\prime}$ in a meaningful fashion, e.g. their ratio diverges like a power law along some subsequence as $p \downarrow p_{c}$, then there really are two correlation lengths - with different exponents. Although the authors consider this to be implausible, there is, at present, no mathematical argument which rules this out. For the purposes of this paper, we will therefore define $\xi^{\prime}$ to be the largest 
number such that, uniformly in $|x|$,

$$
e^{-|x| / \xi^{\prime}} \geqq \frac{1}{e} \tau_{0,|x|}^{\prime}
$$

Thus (in the scaling sense) $\xi^{\prime}$ would be the maximum of $\xi_{a}^{\prime}$ or $\xi_{b}^{\prime}$.

It is worth remarking that, at present, there is no general proof that $\xi^{\prime}$ is finite for $p>p_{c}$ or that $\xi^{\prime}$ diverges as $p \downarrow p_{c}$. A sufficient condition for $\xi^{\prime}<\infty$ is that $p>\hat{\varrho}_{c}$ $[\mathrm{CCN}]^{3}$.

For $p<p_{c}$, a physical definition of a correlation length based on probabilities of finite scale crossing events was introduced [CCF] and exploited in [CCFS]. The finite-size scaling events, which we will denote by $\mathscr{R}_{3, L}$, were easy-way crossings of the rectangles $\Lambda_{3, L}$. It was shown (see e.g. [CC1] or $[\mathrm{CCF}]$ ) that if $\mu_{p}\left[\mathscr{R}_{3, L}\right]$ is too small, there is exponential decay of connectivities. This implies the existence of (universal) constants, $U(d)$, such that if $p>p_{c}, \mu_{p}\left[\mathscr{R}_{3, L}\right] \geqq U(d)$. Furthermore, if $p<p_{c}$, then for any $\lambda \leqq U(d)$, we can define a finite-size scaling correlation length

$$
L_{F, \lambda}(p)=\sup \left\{L \mid \mu_{p}\left[\mathscr{R}_{3, L}\right] \geqq \lambda\right\}
$$

which enjoys the bounds

$$
a(\lambda) L_{F, \lambda}(p) \geqq \xi(p) \geqq \frac{b(\lambda) L_{F, \lambda}}{1+c(\lambda) \log L_{F, \lambda}}
$$

for some nontrivial $a(\lambda), b(\lambda)$, and $c(\lambda)$. Note that as far as critical scaling is concerned, we can identify any $L_{F, \lambda}(p)$ with the correlation length provided that $\lambda \leqq U(d)$. Furthermore, the ratio of two such $L_{F, \lambda}(p)$ 's will diverge (or tend to zero) more slowly than any power of the correlation length.

Above $p_{c}$, a natural object to consider is the maximum scale where one observes crossings by finite clusters:

$L_{F, \kappa}^{\prime}(p)=\sup \left\{L \mid \operatorname{Prob}_{p}\left(\exists\right.\right.$ an easy-way crossing of $\Lambda_{9, L}$ by a finite cluster of bonds) $\geqq \kappa\}$.

Since less is known about the correlation length above threshold, a few remarks are in order.

Remarks. (a) It is clear that if $\kappa$ is chosen foolishly large, $L_{F, \kappa}^{\prime}(p)$ will be zero (or illdefined). However, by choosing $\kappa$ sufficiently small, we can always ensure that, in a region above $p_{c}, L_{F, \kappa}^{\prime}(p)$ exceeds one.

(b) The use of "9" in (53) is for a purely technical reason - stemming from our choice of " 3 " in (51). Our motivation will become clear in the proof of Lemma 6, below; in practice, any number exceeding three would suffice.

(c) A severe disadvantage of these $L_{F}^{\prime}$ is that they are not defined in terms of events which occur in controlled volumes. Thus the results of [CCFS] do not apply directly. On the other hand, a benefit of the $L_{F}^{\prime}$ is that, assuming $P_{\infty}\left(p_{c}\right)=0$, as $p \downarrow p_{c} L_{F, \kappa}^{\prime}(p)$ cannot remain bounded for small enough $\kappa$. Nonetheless, there still remains the danger that the $L_{F}^{\prime}$ are infinite in some intermediate phase above the

\footnotetext{
${ }^{3}$ In fact, for $d>3$, it was shown in [CCN] that $\xi^{\prime}<\infty$ holds under a technically weaker hypothesis
} 
percolation threshold. However, under the usual scaling picture, $L_{F}^{\prime}$ would actually diverge, and do so in a fashion which depends, relatively weakly, on $\kappa$. E.g. for $\kappa$ in some range, the ratio of $L_{F, k}^{\prime}(p)$ to $\xi^{\prime}(p)$ could approach some function $C(\kappa)$.

What we have available at present is, of course, a less robust statement.

Proposition 5. If $\kappa$ is chosen small enough to ensure that $L_{F, \kappa}^{\prime}(p)$ makes sense, then

$$
\xi^{\prime}(p) \geqq \frac{L_{F, \kappa}(p)}{c_{1}(\kappa)+c_{2}(d) \log L_{F, \kappa}(p)}
$$

Proof. If $L_{F}^{\prime}$ and/or $\xi^{\prime}$ is infinite, the bound is seen to be correct. Otherwise, we have, for any $L$,

$\operatorname{Prob}_{p}\left(\Lambda_{L}\right.$ is crossed the easy way by a finite cluster of bonds $) \leqq(e)(9 L)^{2(d-1)} e^{-L / \xi^{\prime}}$.

Substituting in $L_{F, k}^{\prime}(p)$ for $L$, and using the lower bound of $\kappa$ to replace the lefthand side of (54), the result follows after a bit of algebra.

Our principal result will be stated in terms of the $L_{F, \kappa}^{\prime}(p)$. We start with the following estimate:

Lemma 6. Let $p>p_{c}$ be large enough to ensure that $L_{F,{ }_{k}}^{\prime}(p)$ is finite if $\kappa<\frac{1}{6} p_{c} U(d)$. Now, consider the event

$$
\begin{aligned}
\mathscr{G}_{L}= & \left\{\omega \mid \Lambda_{9, L}\right. \text { is crossed the easy way by a path of bonds belonging } \\
& \text { to the infinite backbone. }\} .
\end{aligned}
$$

Then, if $L>L_{F, \kappa}^{\prime}$,

$$
\mu_{p}\left(\mathscr{G}_{L}\right) \geqq(1 / 2) U(d)
$$

Proof. We will examine the event $\mathscr{R}_{3.3 L}$. Start by dividing $\Lambda_{3,3 L}$ into top, bottom and middle thirds which are $\Lambda_{9, L}$ and translates. Denote by $\mathscr{G}_{L}^{(\text {mid) }}$ the translation of the event $\mathscr{G}_{L}$ to the middle copy of $\Lambda_{9, L}$. Obviously,

$$
\mu_{p}\left(\mathscr{R}_{3,3 L}\right)=\mu_{p}\left(\mathscr{G}_{L}\right) \mu_{p}\left(\mathscr{R}_{3,3 L} \mid \mathscr{G}_{L}^{(\mathrm{mid})}\right)+\left(1-\mu_{p}\left(\mathscr{G}_{L}\right)\right) \mu_{p}\left(\mathscr{R}_{3,3 L} \mid\left(\mathscr{G}_{L}^{(\mathrm{mid})}\right)^{c}\right)
$$

Again, underscoring the obvious, it is noted that if the event $\mathscr{G}_{L}^{(\mathrm{mid})}$ fails, in order for the event $\mathscr{R}_{3,3 L}$ to occur, one of two things must happen:

i) Notwithstanding the event $\left(\mathscr{G}_{L}^{\text {(mid) }}\right)^{c}$, the backbone is essential in ensuring that $\Lambda_{3,3 L}$ is crossed.

ii) $\Lambda_{3.3 L}$ is crossed without any help from the backbone.

In the latter case, either a finite cluster or a dangling end has achieved the crossing. As noted previously, in any dangling end there is a particular bond whose removal renders the dangling end a finite cluster. Thus, for all practical purposes, (ii) implies that $\Lambda_{3,3 L}$ is crossed by a finite cluster. An easy calculation shows that possibility (ii) has probability smaller than $\kappa+\frac{1-p}{p} \kappa \leqq \frac{\kappa}{p_{c}}$.

Let us focus on possibility (i). We claim that if the backbone is still essential for $\mathscr{R}_{3,3 L}$ - but does not cross the middle third - then either the bottom or top translate of $\Lambda_{9, L}$ is traversed by a dangling end. Indeed, let us make the observation 
that any two participating backbone bonds are connected to each other by a path inside $A_{3,3 L}$ which consists of participating backbone bonds. Thus, the participating backbone bonds form a cluster which is connected inside $\Lambda_{3.3 L}$. By hypothesis, this cluster cannot have members in both the top and bottom third of the rectangle, so at least one of these must be crossed by a dangling end in order to deliver $\mathscr{R}_{3,3 L}$. We thus have the upper bound of $2 \frac{1-p}{p} \kappa \leqq 2 \frac{\kappa}{p_{c}}$ on the probability of observing (i). Evidently,

$$
\mu_{p}\left(\mathscr{R}_{3,3 L} \mid\left(\mathscr{G}_{L}^{(\mathrm{mid})}\right)^{c}\right) \leqq \frac{3 \kappa}{p_{c}} \leqq(1 / 2) U(d)
$$

so that

$$
\mu_{p}\left(\mathscr{G}_{L}\right) \geqq(1 / 2) U(d) .
$$

The main result will now follow easily.

Theorem 7. Let $\kappa$ be any fixed number which is sufficiently small as described in Lemma 6. Let $\varepsilon$ be chosen so that $p_{c}+\varepsilon$ lies in a region where $L_{F, \kappa}^{\prime}\left(p_{c}+\varepsilon\right)$ is finite. Then there are constants $k_{1}$ and $k_{2}$ such that

$$
\mu_{p_{c-\varepsilon}}\left(\mathscr{R}_{9,2 L_{F}^{\prime}}\right) \geqq k_{1} e^{-k_{2} \varepsilon Q_{\infty}(\varepsilon) L_{F}^{\prime \prime}},
$$

where, in the above, $L_{F}^{\prime}=L_{F}^{\prime}\left(p_{c}+\varepsilon\right)$.

Proof. Consider the rectangle $\Lambda_{9, L}$ with $L$ any length exceeding $L_{F, \kappa}\left(p_{c}+\varepsilon\right)$. By the above Lemma, the event $\mathscr{G}_{L}$ occurs with probability exceeding $(1 / 2) U(d)$. It is easy to see that when $\mathscr{G}_{L}$ does occur, the (conditional) average number of these crossing bonds does not exceed $\frac{9^{(d-1)}}{(1 / 2) U(d)} Q_{\infty}(\varepsilon) L^{d}$. Putting this together with the types of estimates which have been done previously (e.g. the estimates of Theorem 2, with the shortest crossing length, $\ell_{s}$, here replaced by the conditional shortest crossing length, which may be integrated using Jensen's inequality), and setting $L=2 L_{F}^{\prime}$, one obtains the desired result.

Remark. It is obvious that the choices of the increment above and below threshold need not coincide. Thus the previous theorem may be read

$$
\mu_{p_{c}-\varepsilon}\left(\mathscr{R}_{9,2 L_{F\left(\varepsilon^{\prime}\right)}^{\prime}}\right) \geqq k_{1} e^{-k_{2}\left[\varepsilon+\varepsilon^{\prime}\right] Q_{\infty}\left(\varepsilon^{\prime}\right) L_{F}^{\prime} d_{\left(\varepsilon^{\prime}\right)}} .
$$

All the evidence suggests that the most efficient estimates are obtained when $\varepsilon=\varepsilon^{\prime}$ (cf. remarks following previous theorems). However, this device saves us the slight embarrassment that, even if we could be assured that $\xi^{\prime}$ diverged with exponent $v^{\prime}$, how do we know that $v^{\prime}=v$ ?

Corollary. If the critical exponents $v, \beta_{Q}$, and $v^{\prime}$ exist, then

$$
1+\beta_{Q} \leqq \max \left\{d v, d v^{\prime}\right\} \text {. }
$$

Proof. We will start with the weaker assumption that for the appropriate (fixed) choice of $\kappa, L_{F, \kappa}^{\prime}\left(p_{c}+\varepsilon^{\prime}\right) \equiv L_{F, \kappa}^{\prime}\left(\varepsilon^{\prime}\right)$ and $\xi^{\prime}\left(\varepsilon^{\prime}\right)$ diverge along some sequence tending to zero. Using the known result $[\mathrm{G}, \mathrm{CC} 5]$ that below threshold the correlation 
length diverges continuously, we can choose an $\varepsilon\left(\varepsilon^{\prime}\right)$ in such a way that the lefthand side of (58) tends to zero. This implies that

$$
\infty=\lim _{\varepsilon^{\prime} \rightarrow 0}\left[\varepsilon^{\prime}+\varepsilon\left(\varepsilon^{\prime}\right)\right] Q_{\infty}\left(\varepsilon^{\prime}\right) L_{F, \kappa}^{\prime d}\left(\varepsilon^{\prime}\right) .
$$

Now, assuming the existence of critical exponents, we have

$$
1+\beta_{Q} \leqq d v^{\prime}
$$

if $v^{\prime} \geqq v$, or

$$
\frac{v^{\prime}}{v}+\beta_{Q} \leqq d v^{\prime}
$$

if $v^{\prime} \leqq v$. Equations (60) and (61) imply the statement of this corollary.

Remark. As has been discussed previously, we may scale $\varepsilon^{\prime}$ at a much faster rate than $\varepsilon$ to obtain a sub-inequality relating only the quantities defined above threshold. The result, as expressed in critical exponents, is:

$$
(d-1) v^{\prime} \geqq \beta_{Q} .
$$

Equation (62) alone pushes the percolation upper critical dimension beyond five.

\section{Chemical Distance}

The chemical distance is defined (usually above $p_{c}$ ) as the length of the shortest path which connects two regions. For a number of historical and practical reasons, this distance is measured in units of the correlation length. Thus, inherent in any of the usual descriptions of a chemical distance exponent, $\zeta$, is the correlation length itself.

In addition to the polymer physics arena in which $\zeta$ first appeared, the notion of chemical distance plays an important role in a certain class of first passage and random media problems, ${ }^{4}$ as discussed in [RC].

Below, we shall list three plausible (albeit informal) definitions of a $\zeta$, all of which may be identified if one accepts the usual scaling picture of the percolation critical point.

Definition $I$. At $p_{c}$, in rectangles of scale $L$, the shortest crossing between opposing faces, $\ell_{s}(L)$, will scale with $L$ via

$$
\ell_{\delta}(L) \sim L^{\zeta / v^{\prime}} .
$$

\footnotetext{
${ }^{4}$ In an earlier era, the exponent $\zeta$ was believed to be related to the random resistor problem via the relationship $t=(d-2) v+\zeta$, where $t$ is the conductivity exponent (and, presumably, $d \leqq d_{c}$ ). This was based on a picture of "strands" connecting "blobs", and the assumption that the resistance between neighboring blobs is given by their (chemical) separation, while the blobs themselves are relatively perfect conductors.

It is now widely believed that in $d=2, t / v$ is strictly less than one [Z, HDV, HHHS, RAB, LF], while, as we will show here, $\zeta \geqq v$ in any dimension. This necessarily implies a complication of the above "nodes-links" picture - at least in $d=2$. One must now accept the possibility that the intrinsic resistance of the strands has been lowered by internal loops appearing on all scales shorter than the correlation length
} 
Definition II. At $p_{c}+\varepsilon$, in rectangles of scale $\xi^{\prime}(\varepsilon)$, the shortest crossing between opposing faces, $\ell_{0}\left(\xi^{\prime}(\varepsilon)\right)$, scales with $\varepsilon$ via

$$
\ell_{0}\left(\zeta^{\prime}(\varepsilon)\right) \sim \varepsilon^{-\zeta_{\text {II }}} .
$$

Definition $I I I$. At $p_{c}+\varepsilon$, in rectangles of scale $L \gg \xi^{\prime}(\varepsilon)$, the shortest crossings, $\ell_{\diamond}(L)$, between opposing faces will have the scaling

$$
\ell_{s}(L) \sim \frac{L}{\xi^{\prime}(\varepsilon)} 厅(\varepsilon)
$$

with

$$
\sigma(\varepsilon) \sim \varepsilon^{-\zeta_{\mathrm{HI}}} .
$$

Of course, in the above definitions, we have been a little obscure about the sense in which the shortest crossings exhibit this purported scaling, i.e. in I-III do we mean typical or average? According to the usual folklore, this should be inconsequential; as a matter of fact, our results do not distinguish I-III, nor the sense in which they are defined.

Theorem 8. Let $\tilde{\ell}_{0}(L, p)$ denote the median and $\bar{\ell}_{0}(L, p)$ the average shortest-length, easy-way crossing of $\Lambda_{3, L}$ at density $p$, given that one exists. Then

(a) $\mu_{p_{c}-\varepsilon}\left[\mathscr{R}_{3, L}\right] \geqq K e^{-c \varepsilon \tilde{\ell}_{s}\left(L, p_{c}\right)}$,

(a) $\mu_{p_{c}-\varepsilon}\left[\mathscr{R}_{3, L}\right] \geqq K e^{-c \varepsilon \bar{e}_{s}\left(L, p_{c}\right)}$,

or, in general,

(c) $\mu_{p_{c}-\varepsilon}\left[\mathscr{R}_{3, L}\right] \geqq K e^{-c\left[\varepsilon+\varepsilon^{\prime}\right] \tilde{\ell}_{s}\left(L, p_{c}+\varepsilon^{\prime}\right)}$,

(d) $\mu_{p_{c}-\varepsilon}\left[\mathscr{R}_{3, L}\right] \geqq K e^{-c\left[\varepsilon+\varepsilon^{\prime}\right] \bar{t}_{s}\left(L, p_{c}+\varepsilon^{\prime}\right)}$,

where $c$ and $K$ are uniform constants of order unity which may vary from equation to equation.

Proof. These are straightforward applications of the previous techniques used in the proofs of Theorems 2 and 7 and Proposition 4.

Corollary. Provided that critical exponents for the correlation lengths exist, if critical exponents for any of the chemical distances exist, they satisfy

$$
\zeta \geqq v^{\prime}
$$

and

$$
\zeta \geqq \min \left\{1, \frac{v^{\prime}}{v}\right\} .
$$

Remark. We do not consider it a particularly deep result that $\zeta_{\mathrm{I}} \geqq v^{\prime}$. However, it should be noted that the lower bound of $v^{\prime}$ is a better result than $\zeta \geqq 1$ in $d=2$, but is likely to be poorer in higher dimensions.

Proof. We will not exhaust all twelve cases since they all involve only slight modifications of previous techniques. Instead, we will work through a few examples. 
First, observe from (a) or (b) that when $L(\varepsilon) \gtrsim \xi(\varepsilon)$ is chosen to drive $\mu_{p_{c}-\varepsilon}\left[\mathscr{R}_{3, L(\varepsilon)}\right]$ to zero, we obtain

$$
\liminf _{\varepsilon \rightarrow 0} \tilde{l}_{\delta}\left(L(\varepsilon), p_{c}\right)=\infty
$$

and

$$
\liminf _{\varepsilon \rightarrow 0} \varepsilon \bar{\ell}_{\sigma}\left(L(\varepsilon), p_{c}\right)=\infty
$$

which implies $\zeta_{\mathrm{I}} \geqq v / v^{\prime}$.

As for the exponent(s) $\zeta_{\text {III }}$, from (c) and (d), we obtain the inequality

$$
c\left(\varepsilon+\varepsilon^{\prime}\right) \frac{\xi(\varepsilon)}{\xi^{\prime}\left(\varepsilon^{\prime}\right)} \sigma\left(\varepsilon^{\prime}\right) \geqq 1,
$$

where $\lrcorner\left(\varepsilon^{\prime}\right)$ is the quantity described in Definition III, as obtained via an inferior limit of the median or average $L^{-1} \xi^{\prime}\left(\varepsilon^{\prime}\right) \ell_{a}\left(L, p_{c}+\varepsilon^{\prime}\right)$. This gives $\zeta_{\text {III }}>1$ if $v^{\prime} \geqq v$ or $\zeta_{\text {III }} \geqq v^{\prime} / v$ if $v^{\prime} \leqq v$. The derivation for $\zeta_{\text {II }}$ follows the same line of reasoning. It should be noted that the above results are, in essence, independent of the definition of $\xi^{\prime}$, since $\xi^{\prime}$ enters only as a convenient distance scale.

As in the remarks following previous theorems, we may consider the case $\varepsilon=\varepsilon^{\prime a}$ with $a \gg 1$. By driving a to infinity in the resulting exponent inequalities, one obtains that $\zeta_{\mathrm{II}}, \zeta_{\mathrm{III}} \geqq v^{\prime}$.

\section{Concluding Remarks}

It is seen that the bound $\zeta \geqq 1$ (modulo the provisos) is the second half of any of the earlier inequalities (1), (2), or (3). The first halves amount to upper bounds on the various versions of $\zeta$, some of which were implicit in [CC1, CC2]. Thus, from Theorem 2 one can show that:

$$
\zeta_{\text {III }} \leqq f-\beta_{Q}+v^{\prime}
$$

and from Proposition 4:

$$
\zeta_{\text {III }} \leqq \mu-\beta_{Q}+v^{\prime}
$$

Furthermore, examining the considerations of Sect. 4, one has

$$
\zeta_{\mathrm{II}} \leqq d v^{\prime}-\beta_{Q} .
$$

If one accepts the standard scaling picture, all of these results are equivalent below $d_{c}$. Above $d_{c}$ [with $d$ replaced by $d_{c}$ in (68)] these constitute mean-field bounds. It is worth noting that, according to the numerical work of [PS], these upper bounds on $\zeta$ are reasonably good in low dimension.

Acknowledgements. The authors would like to thank H. Kesten for countless enjoyable and informative discussions. In particular, H.K. deserves more than thanks for a marathon session during which many of the results of Sect. 5 were derived.

We would like also like to thank A.H.C. (L.M.) for suggesting that we undertake this project. 


\section{References}

[A] Aizenman, M.: Geometric analysis of $\varphi^{4}$ fields and Ising models. Parts I and II. Commun. Math. Phys. 86, 1-48 (1982)

[AB] Aizenman, M., Barsky, D.J.: Sharpness of the phase transition in percolation models. Commun. Math. Phys. 108, 489-526 (1987)

[ACCFR] Aizenman, M., Chayes, J.T., Chayes, L., Fröhlich, J., Russo, L.: On a sharp transition from area law to perimeter law in a system of random surfaces. Commun. Math. Phys. 92, 19-69 (1983)

[AF] Aizenman, M., Fernández, R.: On the critical behavior of the magnetization in highdimensional Ising models. Rutgers University preprint (1986)

[AG] Aizenman, M., Graham, R.: On the renormalized coupling constant and the susceptiblity in $\varphi_{4}^{4}$ field theory and the Ising model in four dimensions. Nucl. Phys. B 225 [FS 9], 261-288 (1983)

[AKM] de Alcantara Bonfim, O.F., Kirkham, J.E., McKane, A.J.: Critical exponents for the percolation problem and the Yang-Lee edge singularity. J. Phys. A: Math. Gen. 14, 2391-2413 (1981)

[AKN] Aizenman, M., Kesten, H., Newman, C.M.: Uniqueness of the infinite cluster and continuity of the connectivity functions for short and long range percolation. Commun. Math. Phys. (to appear)

[AN] Aizenman, M., Newman, C.M.: Tree graph inequalities and critical behavior in percolation models. J. Stat. Phys. 36, 107-143 (1984)

[BH] Broadbent, S.R., Hammersley, J.M.: Percolation processes. I. Crystals and mazes. Proc. Camb. Phil. Soc. 53, 629-641 (1957)

[CC1] Chayes, J.T., Chayes, L.: Percolation and random media. In: Critical phenomena, random systems and gauge theories. Proceedings of the Les Houches Summer School, Session XLIII, pp. 1001-1142. Osterwalder, K., Stora, R. (eds.) Amsterdam: Elsevier 1986

[CC2] Chayes, J.T., Chayes, L.: Bulk transport properties and exponent inequalities for random resistor and flow networks. Commun. Math. Phys. 105, 133-152 (1986)

[CC3] Chayes, J.T., Chayes, L.: Inequality for the infinite cluster density in Bernoulli percolation. Phys. Rev. Lett. 56, 1619-1622 (1986)

[CC4] Chayes, J.T., Chayes, L.: The mean field bound for the order parameter of Bernoulli percolation. In: Percolation theory and ergodic theory of infinite particle systems, pp. 49 71. Kesters, H. (ed.). Berlin, Heidelberg, New York: Springer 1987

[CC 5] Chayes, J.T., Chayes, L.: Critical points and intermediate phases on wedges of $\mathbb{Z}^{d}$. J. Phys. A: Math. Gen. 19, 3033-3048 (1986)

[CCF] Chayes, J.T., Chayes, L., Fröhlich, J.: The low-temperature behavior of disordered magnets. Commun. Math. Phys. 100, 399-437 (1985)

[CCFS] Chayes, J.T., Chayes, L., Fisher, D.S., Spencer, T.: Finite-size scaling and correlation length bounds for disordered systems. Phys. Rev. Lett. 57, 2999-3002 (1986)

[CCN] Chayes, J.T., Chayes, L., Newman, C.M.: Bernoulli percolation above threshold: an invasion percolation analysis. Ann. Probab. (1987)

[CCGKS] Chayes, J.T., Chayes, L., Grimmett, G.R., Kesten, H., Schonmann, R.H.: In preparation

[F] Fröhlich, J.: On the triviality of $\lambda \varphi_{i}^{4}$ theories and the approach to the critical point in $d>4$ dimensions. Nucl. Phys. B 200 [FS 4], 281-296 (1982)

[FS] Fröhlich, J., Sokal, A.: Unpublished (1984)

[FSS] Fröhlich, J., Simon, B., Spencer, T.: Infrared bounds, phase transitions and continuous symmetry breaking. Commun. Math. Phys. 50, 79-85 (1976)

[G] Grimmett, G.R.: Bond percolation on subsets of the square lattice, and the threshold between one-dimensional and two dimensional behaviour. J. Phys. A: Math. Gen. 16. 599-604 (1983)

[GS] Gaunt, D.S., Sykes, M.F.: Series study of random percolation in three dimensions. J. Phys. A: Math. Gen. 16, 783-800 (1983) 
[HDV] Hermann, H.J., Derrida, B., Vannimenus, J.: Superconductivity exponents in twoand three-dimensional percolation. Phys. Rev. B 30, 4080-4082 (1984)

[HHHS] Hong, D.C., Havlin, S., Hermann, H.J., Stanley, H.E.: Breakdown of AlexanderOrbach conjecture for percolation: exact enumeration of random walks on percolation backbones. Phys. Rev. B 30, 4083-4086 (1984)

[K 1] Kesten, H.: Scaling relations for 2D-percolation. IMA preprint (1986)

[K2] Kesten, H.: First-passage percolation and a higher dimensional generalization. Contemp. Math. 41, 235-251 (1985)

[K 3] Kesten, H.: Surfaces with minimal weight and maximal flows: A higher dimensional version of first-passage percolation, III. J. Math. (1987)

[LF] Lobb, C.J., Frank, D.J.: Percolative conduction and the Alexander-Orbach conjecture in two dimensions. Phys. Rev. B 30, 4090-4092 (1984)

[MMS] Menshikov, M.V.: Doklady Akad. Nauk. C.C.C.P. 288, 1308-1311 (1986) (in Russian)

Menshikov, M.V., Molchanov, S.A., Sidovenko, A.F.: Itogi Nauki i Techniki (Series of Probability Theory, Mathematical Statistics, Theoretical Cybernetics) 24, 53-110 (1986) (in Russian)

[PL] Priest, R.G., Lubensky, T.C.: Critical properties of two tensor models with applications to the percolation problem. Phys. Rev. B 13, 4159-4171 (1976) (Erratum 14, 5125)

[PS] Pike, R., Stanley, H.E.: Order propagation near the percolation threshold. J. Phys. A: Math. Gen. 14, L169-L177 (1981)

[RAB] Rammal, R., Angeles d'Auriac, J.C., Benoit, A.: Universality of spectral dimension of percolation clusters. Phys. Rev. B 30, 4087-4089 (1984)

[RC] Ritzenberg, A.L., Cohen, R.J.: First-passage percolation: Scaling and critical exponents. Phys. Rev. B 30, $4038-4040$ (1984)

[Z] Zabolitsky, J.G.: Monte Carlo evidence against the Alexander-Orbach conjecture for percolation conductivity. Phys. Rev. B 30, 4077-4079 (1984)

Communicated by A. Jaffe

Received May 7, 1987

Note added in proof. Since the submission of this paper, issues (a) and (b) discussed in the paragraphs below Eq. (45) have been settled. In particular, the limits of $n^{-1} \log \tau_{0, n}^{\prime}$ and $n^{-1} \log \tau_{0, n}^{\prime \prime}$ exist (and agree), and an above threshold bound of the form (46), with power law modifications, has been established. The latter implies that $\xi_{a}^{\prime}=\xi_{b}^{\prime}$, as defined in Eqs. (47) and (49). However, the question of nontriviality for all $p>p_{c}$ remains open. See [CCGKS] for details. 